\title{
Correction to: Development of a software for mobile devices designed to help with the management of individuals with neglected tropical diseases
}

\author{
Priscilla Elias Ferreira da Silva ${ }^{1}\left[\right.$. Gerson dos Santos Fonseca Junior ${ }^{1} \cdot$ Roberta Bianchi Ambrozio $^{1}$. \\ Monique Gomes Salles Tiburcio ${ }^{2}$. Gabrielly Borges Machado ${ }^{3}$. Silvio Fernando Guimarães de Carvalho ${ }^{4}$. \\ Edward José de Oliveira $^{5} \cdot$ Luciana de Almeida Silva Teixeira $^{1} \cdot$ David Calhau Jorge $^{1}$
}

Published online: 20 August 2021

(c) Sociedade Brasileira de Engenharia Biomedica 2021

Correction to: Research on Biomedical Engineering (2020) 36:527-537

https://doi.org/10.1007/s42600-020-00090-8

The authors apologise for forgetting to mention the following financial support in the article: The study was funded by Fundação de Amparo à Pesquisa do Estado de Minas Gerais (FAPEMIG), APQ-01608-16.

Publisher's note Springer Nature remains neutral with regard to jurisdictional claims in published maps and institutional affiliations.

The original article can be found online at https://doi.org/10.1007/ s42600-020-00090-8.

Priscilla Elias Ferreira da Silva

priefsilva@gmail.com

Gerson dos Santos Fonseca Junior gersonmania@gmail.com

Roberta Bianchi Ambrozio robertaambrozio@gmail.com

Monique Gomes Salles Tiburcio monique_tiburcio@yahoo.com.br

Gabrielly Borges Machado gabyborges100@hotmail.com

Silvio Fernando Guimarães de Carvalho guimaraescarvalho@yahoo.com.br

Edward José de Oliveira edward.oliveira@ fiocruz.br
Luciana de Almeida Silva Teixeira lalmeidast@gmail.com

David Calhau Jorge david.jorge@uftm.edu.br

1 Universidade Federal do Triângulo Mineiro (UFTM) Uberaba, Av. Getúlio Guaritá, s/n, Uberaba, MG, Brazil

2 Universidade Federal de Lavras (UFLA) Lavras, Lavras, Brazil

3 Faculdade de Medicina Athenas, Paracatu, Brazil

4 Universidade Estadual de Montes Claros (Unimontes) Montes Claros, Montes Claros, Brazil

5 Instituto René Rachou - Fiocruz Minas, Belo Horizonte, Minas Gerais, Brazil 